\title{
Maternal health care utilization and neonatal mortality in Nigeria: looking beyond the micro-level pathway of influence
}

\author{
Ikeola Adeoye ${ }^{1}$, Gbenga Quadri ${ }^{1}$, Sunday A. Adedini ${ }^{2,3}$, \\ ${ }^{1}$ Department of Epidemiology and Medical Statistics, Faculty of Public Health, College of Medicine, \\ University of Ibadan, Ibadan, Nigeria \\ ${ }^{2}$ Department of Demography and Social Statistics, Faculty of Social Sciences, \\ Obafemi Awolowo University, Ile-Ife, Nigeria \\ ${ }^{3}$ Demography and Population Studies Programme, Schools of Public Health and Social Sciences, \\ University of the Witwatersrand, Johannesburg, South Africa \\ adeoyeikeola@yahoo.com, iadeoye@cartafrica.org
}

\begin{abstract}
Neonatal mortality is a neglected but largely preventable public health challenge in Nigeria. The country contributes the largest number of neonatal deaths in Africa, and this is an important reason for the failure of the country to meet Millennium Development Goal (MDG4) of reducing child mortality. Maternal health services provide the platform for delivering cost-effective interventions that reduce maternal and child mortality. Thus, we examined the relationship between the utilization of maternal health services and neonatal mortality in Nigeria by carrying out a multilevel Cox proportional regression analysis of the most recent Nigeria Demographic and Health Survey (2013 NDHS) in order to decompose the micro and macro level factors on the pathway of influence for neonatal mortality. Hazards of neonatal death were significantly lower for children whose mothers had 4 or more antenatal visits by skilled providers (HR: $0.78, \mathrm{Cl}: 0.6 \mathrm{I}-0.98, \mathrm{p}<0.05)$ and whose mothers received postnatal care from skilled provider (HR: $0.4 \mathrm{I}, \mathrm{Cl}: 0.30-0.56, \mathrm{p}<0.05)$ even after adjusting for other control variables. There was also a significantly elevated hazards of neonatal mortality for mothers in rural areas (HR: I.44, Cl: I.09-I.90). Our findings highlight the importance of maternal health care services for neonatal mortality reduction especially in the rural areas.
\end{abstract}

Keywords: Neonatal mortality, maternal health care, multilevel analysis, continuum of care, Nigeria,

\section{Introduction}

Neonatal mortality, death occurring within the first 28 days of life, has become a global public health concern with an estimated 4 million neonatal deaths annually (Lawn et al, 2005; Martines J, 2005; UNICEF, 20I I). Unfortunately, this global burden is disproportionately borne by developing countries in South East Asia and sub Saharan Africa (Chowdhury et al, 2005; Shiffman J, 2010). The failure to achieve the fourth Millennium Development Goal (MDG-4) which aimed at reducing under-five mortality by two thirds by the year 2015 was partly because of persistent neonatal deaths (United Nations 2010, Oestergaard, 20II). Globally about $41 \%$ all underfive mortality is attributable to neonatal deaths and most of these occur in the first week of life (WHO, 2009). Nigeria contributes significantly to the global neonatal burden (estimated 280,073 deaths annually) having the third highest figures after India and China and bearing the largest burden in Africa. Although considered one of the largest economies in Africa, countries (Uganda, Malawi, Eritrea, Tanzania) with lower Gross National Income (GNI) per capital in the continent report much lower neonatal rates (Oestergaard, 20I I). In addition, the latest NDHS report (20I3) shows that there are wide disparities in neonatal mortality rates within the country by wealth status, region and residence (NPC, Macro, 20I4).

The health of the mother and the newborn are closely linked (UNICEF, 20II) implying that conditions in the mother can adversely affect neonatal survival. At the same time, the direct causes of neonatal deaths which account for most NMM are preterm birth (28\%), severe infections $(26 \%)$, birth asphyxia (23\%) and neonatal tetanus (7\%) (Liu et al, 2012). Fortunately these conditions are preventable by simple, cost-effective interventions that can be provided through maternal health services (Darmstadt, 2005). Maternal health services can provide a continuum of care for the mother and newborn from pregnancy to childbirth and the post- 
delivery periods through antenatal care, skilled birth attendance and postnatal care (Marsh, 2002).

Antenatal care aims to optimize the health of the mother and the child. ANC by skilled attendants can provide health interventions that can enhance neonatal survival; through the provision of accurate health information and disease prevention. For instance, the provision of tetanus toxoid immunization, early detection and management of pregnancy complications like antepartum haemorrhage and pre-eclampsia. Despite the fact that ANC coverage is high in several African countries, the content and the quality of care varies within different health systems. Apparently, ANC has a direct impact on the maternal health, the direct effect on the newborn is not as recognized, however a Cochrane review has reported that antenatal steroids improves the survival of premature newborns (Mwansa-Kambafwile J et al, 2010). Importantly, studies have shown that obtaining ANC from skilled attendants facilitates delivery with skilled attendants (Acuin CS et al 20I I; Kolola T et al, 2016)

Skilled attendance (SA) at birth is one of the most important strategies for reducing maternal and neonatal mortality (WHO, 20I3) Unfortunately most NNM takes place at home in the absence of skilled attendants (UNICEF, 20II). The WHO (2005) defines a skilled attendant as an individual (doctor, nurse or midwife) who is trained to proficiency in the skills needed to manage normal pregnancy, childbirth and immediate postpartum period, and in the identification, management and referral of complications in women and newborn. Unfortunately the coverage of skilled attendance in Nigeria is still low. The last NDHS report (20I3) documented that only $38 \%$ of the deliveries that took place five years preceding the survey were supervised by skilled attendants. SA at delivery is crucial because of the provision of essential obstetric and newborn services. However there are variations in the levels of skills and competence of SA. In addition, lack of health infrastructure and weak health systems hinder the provision of quality services by skilled attendants especially in sub-Saharan Africa. Singh and coworkers (2013) assessed the influence of SA on neonatal mortality reduction across three continental regions - Latin America/Caribbean, South East Asia and Africa. They found that although SA was protective for NNM in Latin America it was associated with an increase risk in Africa. They explained that SA in Africa is faced with more challenges in the provision of care during childbirth, such as handling complicated cases that had been mismanaged by unskilled attendants, having questionable levels of the skills and competence as well as working with inadequate health infrastructure (Singh et al, 20l3).

Postnatal care is the integrated care provided for the mother and the new born within the first 6 weeks after delivery (WHO 20I3). However, it is the most ignored of the three components of maternal health services. The essential services provided in PNC for improved neonatal survival include promotion of immediate and exclusive breastfeeding, clean cord care, maintenance of body temperature e.g. Kangaroo care. Experts have noted that early contact is important for neonatal survival. The WHO recommends that babies should been seen in less than 48 hours after delivery. For example in Bangladesh early postnatal care is considered a national priority (Winch, 2005).

In Nigeria, neonatal mortality is of public health importance; 37 per 1000 live births are dying within the first 28 days of life (NDHS 2013). Even the post MDG era, tackling neonatal mortality remains a priority in order to reduce child mortality. Currently, emphasis needs to be placed on NNM reduction (which had been previously neglected) rather than infant mortality or under-five mortality which have received more attention and achieved more success in Nigeria. For example the Nigeria DHS report indicated that post neonatal mortality rates have been declining faster than NNM rates in the country while NNM rate was 48 per 1000 in 2003 and 37//000 in 2013; post neonatal mortality rates dropped from $52 / 1000$ to $31 / 1000$ within the same period. In addition while $48 \%$ of infant mortality rate occurred in the neonatal period in 2003, this proportion increased to $54 \%$ by 2013 . However, there paucity of data that can inform policy and programmes on neonatal mortality reduction in Nigeria. Earlier studies had used hospital based data (Owa J.A. \& Osinaike, 1998; Suleman and Mukuolu, 2014), a few studies have begun to emerge using a nationally representative data - Ezeh et al 2014, Akinyemi et al, 2015 both of which explored the determinants of NNM in Nigeria. Adewuyi and colleagues (2017) investigated the rural-urban differences in the determinants of NNM. Our study goes further to investigate the effect of the utilization of maternal health services and neonatal mortality in Nigeria using a multilevel approach in the secondary analysis of the NDHS data of 2013.

\section{CONCEPTUAL FRAMEWORK}

The conceptual framework used for this study is adapted from the Mosley-Chen Framework. Mosley and Chen (1984) developed an analytical framework for the study of child survival in developing countries. The framework incorporates biological (proximate) and socio-economic determinants of child survival 
through the integration of techniques in the medical and social sciences. The framework also posits that the determinants of child mortality could be measured at different hierarchical levels, including individual, household and community levels. In the present study, selected variables at the individual level include maternal factors, paternal characteristics, neonatal factors and the health systems factors. The selected health service factors are antenatal care utilization, type of birth attendants and place of delivery. These health-related factors also fall under the proximate determinant of personal illness control enshrined in the Mosley-Chen Framework. Marsh et al (20II) also further elaborates on the continuum of care and the constellation of public health interventions that can improve maternal and neonatal survival. This was also considered in this work.

Further, we employed multilevel model approach in this study, as Mosley-Chen Framework acknowledges that childhood mortality is a function of layers of determinants - ranging from distal or community level to individual level or socioeconomic factors, and to the proximate determinants (i.e. more direct variables). The community level variables considered in this study include region, place of residence, community poverty, and community level of education. The utility of these variables as predictors of child survival has been established in previous studies (Adedini et al., 2015; Antai, 20l I; Omariba et al., 2007).

\section{DATA AND METHODS \\ Data Source}

This study is a secondary data analysis of the nationally representative Nigeria Demographic Health Survey 2013. The survey was conducted by the National Population Commission (NPC), Nigeria in conjunction with the ICF International USA, in 36 states and the Federal Capital Territory of Nigeria. The sampling frame was from the list of enumeration areas (EAs) prepared for the 2006 Population Census of the Federal Republic of Nigeria and provided by the National Population Commission. For this particular study, the kids recode data in which the birth outcome of 31,428 reproductive age women aged 15-49 women from both urban and rural areas in Nigeria was used. These women had experienced child birth 5years preceding the survey. The data was weighted to account for the sampling variability resulting from greater respondents from rural compared to the urban areas. Permission to use the 2013 NDHS data for this study was obtained from the ICF International - the agency responsible for the conduct of Demographic and Health Surveys globally

\section{Variable Definition}

Outcome Variable: - Neonatal mortality defined as the probability of dying within the first 28 days of life. The variable was dichotomized where death from day I to the 28th day was recoded to I "success" and those that survived after the 28th day to 5years was recoded to 0 "failure".

Explanatory Variables: - These variables were derived from the Child Survival Framework by Mosley and Chen (1984) which included Individual and Proximate level. The conceptual framework for Advancing Newborn Health and Survival in developing countries by Marsh and co-workers (2002) which described the essential elements of routine maternal and new born care at critical periods necessary for survival. The primary independent variables were maternal use of antenatal, delivery and postnatal health care services. In addition, the other variables included the individual level determinants which included maternal age, parity, maternal occupation, maternal education, breast feeding and preceding birth interval, place of residence and region of the country.

\section{Definition of community level variables}

The variables of interest at the community-level include: region of residence, place of residence, community poverty level and community level of education. Region of residence has six categories (i) North-central (ii) North-east (iii) North-west (iv) South-east (v) South-south and (vi) South-west. Place of residence is categorized as (i.) urban and (ii) rural. Community poverty level and community level of education were generated by aggregating the individual level wealth quintile and individual level of education, respectively. This aggregation was done at the level of primary sampling units (PSU). PSU served as a cluster or community in the 2013 NDHS and 904 clusters were sampled for the survey across the country. The variable - community poverty which was generated at the level of the PSU was later categorized as (i) rich (ii) middle (iii) rich; while community education was also divided into tertile i.e. (i) community with low concentration of women with at least secondary education (ii) medium and (iii) community with high concentration of women with at least secondary education. 
Table 1: Variables Definitions

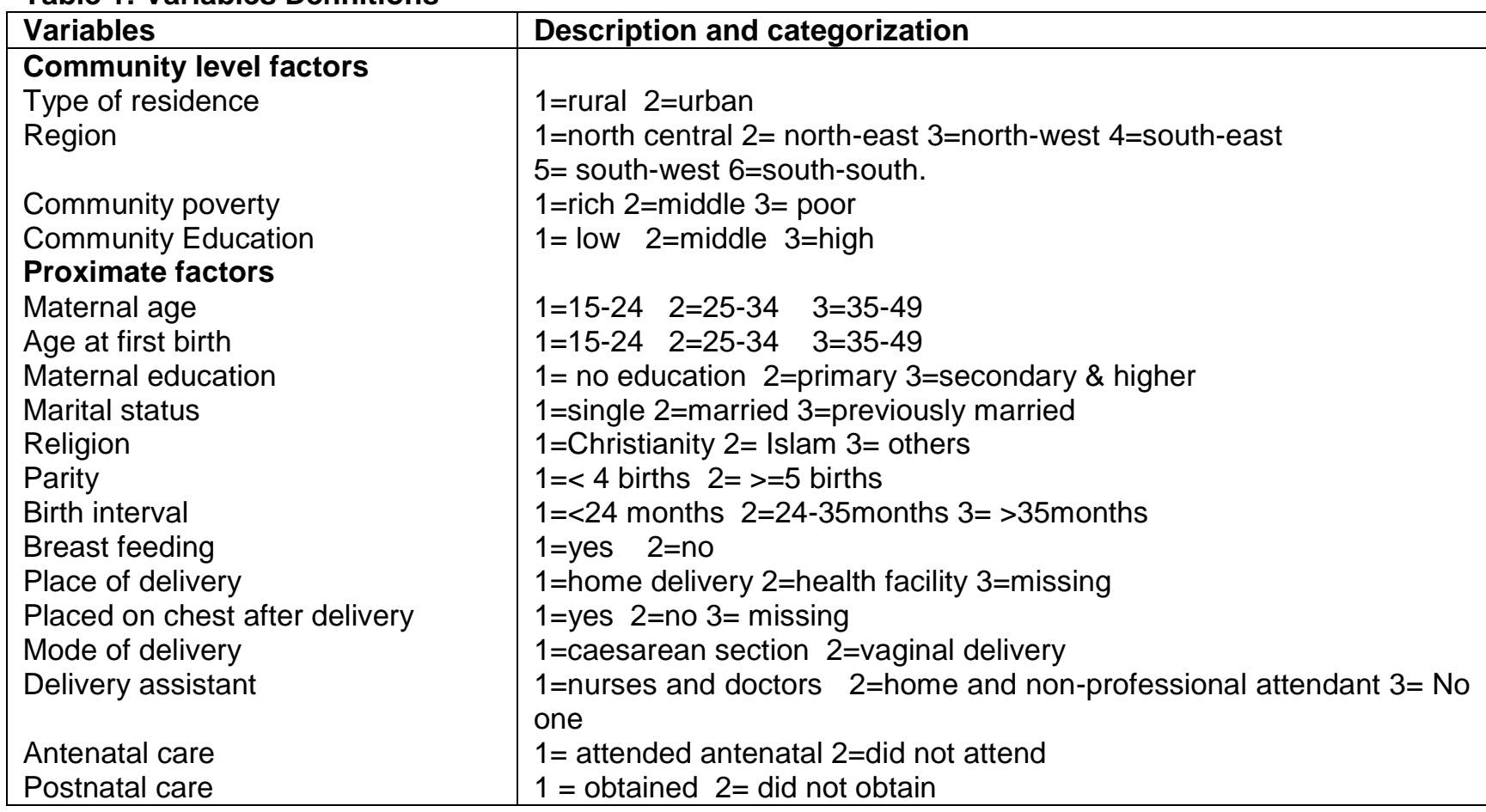

\section{Data Analysis}

Descriptive and inferential statistics were employed in the analysis of the data. The background characteristics of the study population is described using frequency distribution of women age $15-49$ who had a live birth in the five years preceding the survey. The neonatal mortality rates (per 1000) and $95 \%$ confidence intervals were calculated by certain individual maternal, contextual, neonatal characteristics. Bivariate Analysis: Chi-square test was also used to explore the association between the utilization of maternal health services and neonatal maternal. Multivariate analysis: Multilevel Cox proportional hazards model was employed to examine association between neonatal mortality and selected variables across individual maternal and contextual characteristics. All analysis was done using Stata (version II.I). The multilevel cox regression analysis was performed using the generalized linear latent and mixed models (GLLAMM) procedure downloadable and implementable in Stata.

\section{RESULTS}

Table 2 describes the background characteristics of mothers who had a live birth in the five years preceding the survey by the socio-demographic characteristics and their utilization of maternal health care services. Most of the mothers were less than 35 years $(65.1 \%), 46.9 \%$ had no education, $97.8 \%$ were married, $59.1 \%$ had 4 or less number of children, $45.9 \%$ were poor belonging to the lowest wealth tertile and $67.1 \%$ dwelt in the rural areas. The distribution of the women by geopolitical zone is shown as follows: north central (14.7\%), north east http://aps.journals.ac.za
(20.7\%), north west (3I.5\%), south east $(8.9 \%)$, south-south (II.9\%), south west (I2.3\%). Similarly, $43.1 \%$ received antenatal care of which $33.3 \%$ had at least 4 ANC visits, less than half (4I.1\%) delivered with skilled provider and $18.3 \%$ received postnatal care.

Table 3 shows neonatal mortality rates and $95 \%$ confidence intervals by individual maternal contextual and neonatal characteristics. The highest NMR per 1000 were reported among mothers in the youngest age group $47.9(2.5-53.1)$, not currently married 49.6 (39.1 - 45.3), grand multiparous 41.5 (39.1 47.4), rural dwellers $4 I . I(39 . I$ - 4I.3), women living in the North-west geopolitical region 45.5 (4I.3 50.0). Others were birth interval of less than 24 months from last delivery and poor women. The neonatal mortality rate by certain neonatal characteristics are shown, and the neonatal mortality rates are higher among male infants 42.7(39.7 46.0), and birth order greater than 6, 47.7(42.1 53.7), and smaller sized babies $99.1(6.8-1 \mid 3)$,

Table 4 shows the association between the utilization of maternal health services along the continuum of care and neonatal mortality. Significantly higher proportions $(p<0.05)$ of women who had caesarean operation $(78.7 \%)$ were attended by skilled provided during ANC (5I.4\%), delivery $(50.4 \%)$ and PNC (56.3\%) reported higher proportion of neonatal deaths. However, early initiation of breast feeding was associated with lower occurrence of neonatal deaths (34.2\%).

Results from the multilevel Cox proportional regression analysis are presented in Tables 5 and 6 . Eight multilevel models were fitted in all. Four 
models were fitted to examine the influence of antenatal care utilization and selected control variables on neonatal death, and four models examined the effects of postnatal care utilization and selected covariates on neonatal mortality. Model 0 in both Tables 5 and 6 contained no explanatory variables, but was fitted to decompose the total variance into its individual and community level components, and to validate the use of multilevel analysis in the paper. Model I examined the effects of maternal health care utilization (ANC - Table 5; and PNC - Table 6) on neonatal death, while also adjusting for the effects of selected individual level variables. Model 2 achieved similar aim, but also adjusted for community level variables. Model 3 is the full model which incorporated all the selected variables into the multilevel analysis.

As presented in Model 0 (Tables 5 and 6), the results from the null model showing a significant random effects indicate that the intercepts for the Cox regression model are significant across communities, hence the appropriateness of accounting for the hierarchical structure of the data using multilevel analysis. As shown in Models I-3 (Table 4), hazards of neonatal death were significantly lower for children whose mothers had 4 or more antenatal visits by skilled providers (Modell: HR: 0.76, Cl: 0.6I-0.95, p<0.05; Model 2: HR: 0.80, Cl: 0.66-0.97, p<0.05; Model 3: HR: 0.78, Cl: 0.6I-0.98, $\mathrm{p}<0.05)$ compared to their counterparts whose mothers had less than 4 visits. These results consistently showed the protective advantage of antenatal care utilization for child survival during the neonatal period, even after adjusting for the effects of selected individual- and community-level variables. Results of fixed effects (Model 3) also showed a significantly lower risks of neonatal mortality for children with preceding birth interval of 2 years or higher (HR: 0.75, Cl:0.58-0.96, $\mathrm{p}<0.05$ ), and children of parity 3-4 ((HR: $0.37, \mathrm{Cl}: 0.29-0.47)$ compared to children in the reference categories. Results from full model further revealed a significantly elevated hazards of neonatal mortality for children of mothers aged 35 years or older (HR: 3. I5, Cl: 2.04-4.88), and children of mothers in rural areas (HR: I.44, Cl: I.09I.90) compared to children in the reference groups.

The results examining the effects of postnatal care utilization and other selected control variables (as shown in Table 6) indicated a significantly lower risks of neonatal mortality for children whose mothers received postnatal care from skilled provider (Model I: HR: 0.40, Cl: 0.29-0.54, p<0.05; Model 2: HR: 0.35, Cl: 0.27-0.47, p<0.05; Model 3: HR: 0.4I, $\mathrm{Cl}: 0.30-0.56, p<0.05)$. The protective effect of postnatal care uptake on child survival was reaffirmed after adjusting for the effects of possible confounding variables. Also, children with higher birth interval (HR: 0.66, Cl: 0.50-0.86, $\mathrm{p}<0.05$ ); and those of parity 3-4 (HR: 0.38, Cl: 0.29-0.48, p<0.05) had significantly lower risks of neonatal death than their counterparts in the reference categories. Further, elevated hazards of neonatal death were established for children whose mothers were aged 35 years or older (HR: 3.46, Cl: 2.20-5.44, $\mathrm{p}<0.05$ ), and children of mothers in rural areas (HR: I.43, Cl: I.07-I.90, $\mathrm{p}<0.05)$.

Goodness of fit of the multilevel models was determined in this study using Akaike information criterion (AIC) and Bayesian information criterion (BIC). As shown in Tables 5 and 6, the lower values of $\mathrm{AIC}$ and $\mathrm{BIC}$ indicate a better fit.

Table 2: Background characteristics of study participants by socio-demographic characteristics and maternal health care utilization.

\begin{tabular}{ccc}
\hline Characteristics & Frequency & Percentage \\
\hline Antenatal Care & & 43.00 \\
Received Antenatal Care & & 57.00 \\
Yes & 13530 & 33.31 \\
No & 17952 & 9.67 \\
Number of Visits & & 10,487 \\
$\quad$ Less than 4 & 3043 & 40.98 \\
Delivery Care & & 50.46 \\
Type of Attendant at birth & 12900 & 12.14 \\
Skilled attendant & 15887 & $(2.14)$ \\
$\quad$ Non Skilled & 3,822 & $(97.86)$ \\
No one & & \\
Mode of delivery & 659 & 18.28 \\
Caesarean Section & 30823 & 81.72
\end{tabular}


African Population Studies Vol 3I, No I,(Supp.), 2017

\begin{tabular}{|c|c|c|}
\hline Immediate & 30086 & (95.73) \\
\hline Not immediate & 1396 & $(4.40)$ \\
\hline \multicolumn{3}{|l|}{$\begin{array}{c}\text { Socio-demographic } \\
\text { Maternal Age }\end{array}$} \\
\hline $15-24$ & 7614 & (24.19) \\
\hline $25-34$ & 15698 & $(40.86)$ \\
\hline$\geq 35$ & 8170 & (25.59) \\
\hline \multicolumn{3}{|l|}{ Education } \\
\hline No Education & 14762 & $(46.89)$ \\
\hline Primary & 6432 & (20.43) \\
\hline Secondary and above & 10288 & (32.68) \\
\hline \multicolumn{3}{|l|}{ Marital Status } \\
\hline Married & 29990 & (95.26) \\
\hline Not Married & 1492 & $(4.74)$ \\
\hline \multicolumn{3}{|l|}{ Religion } \\
\hline Christianity & 12654 & $(40.19)$ \\
\hline Islam & 18354 & $(58.30)$ \\
\hline Others & 474 & $(1.51)$ \\
\hline \multicolumn{3}{|l|}{ Parity } \\
\hline$\leq 4$ & 18590 & $(59.15)$ \\
\hline 5 and above & 12892 & $(40.85)$ \\
\hline \multicolumn{3}{|l|}{ Place of Residence } \\
\hline Urban & 21131 & $(67.12)$ \\
\hline Rural & 10351 & (32.88) \\
\hline \multicolumn{3}{|l|}{ Geographical Zone } \\
\hline North Central & 4614 & $(14.66)$ \\
\hline North East & 6517 & (20.70) \\
\hline North West & 9906 & (31.47) \\
\hline South East & 2816 & $(8.94)^{\prime}$ \\
\hline South South & 3747 & (11.90) \\
\hline South West & 3882 & (12.33) \\
\hline \multicolumn{3}{|l|}{ Wealth Index } \\
\hline Poor & 14462 & $(45.94)$ \\
\hline Average & 6272 & (19.92) \\
\hline Rich & 10748 & (34.14) \\
\hline
\end{tabular}


Table 3: Neonatal mortality rates and $95 \%$ confidence interval by individual maternal, contextual characteristics and neonatal factors.

\begin{tabular}{|c|c|c|c|}
\hline Maternal factors & $\begin{array}{ll}\begin{array}{l}\text { Total } \\
\text { births }=31428\end{array} & \text { live } \\
\end{array}$ & $\begin{array}{l}\text { Neonatal mortality } \\
1189(\%\end{array}$ & NMR(95\%Cl) \\
\hline \multicolumn{4}{|l|}{$\begin{array}{l}\text { INDIVIDUAL } \\
\text { Maternal age }\end{array}$} \\
\hline $15-24$ & 7614 & $342(28.76)$ & $47.9(2.5-53.1)$ \\
\hline $25-34$ & 15698 & $516(43.40)$ & $32.9(1.4-35.7)$ \\
\hline \multicolumn{4}{|l|}{ Education } \\
\hline No education & 14762 & $593(49.87)$ & $40.2(37.1-44.3)$ \\
\hline Primary & 6432 & $274(23.04)$ & $42.6(37.8-40.4)$ \\
\hline \multicolumn{4}{|l|}{ Marital status } \\
\hline Married & 29990 & $1115(93.78)$ & $37.2(35.1-42.1)$ \\
\hline $\begin{array}{l}\text { Not married } \\
\text { Parity }\end{array}$ & \multicolumn{2}{|c|}{ Parity } & $49.6(39.1-45.3)$ \\
\hline 1 & 3624 & $135(11.35)$ & $37.1(31.2-40.3)$ \\
\hline $2-4$ & 14966 & $519(43.65)$ & $34.7(31.8-37.3)$ \\
\hline \multicolumn{4}{|l|}{$\begin{array}{l}>0 \mathrm{Or}=5 \\
\text { Birth interval }\end{array}$} \\
\hline$<24$ & 5817 & $314(26.41)$ & $54(48.3-52.4)$ \\
\hline $24-35$ & 9868 & $305(25.65)$ & $30.9(27.6-35.5)$ \\
\hline$>35$ & 15797 & $570(47.94)$ & $36.1(33.2-34.7)$ \\
\hline \multicolumn{4}{|c|}{ CONTEXTUAL FACTORS } \\
\hline Urban & 10351 & $319(26.83)$ & $30.8(27.5-31.5)$ \\
\hline Rural & 21131 & $870(73.17)$ & $41.1(38.5-41.3)$ \\
\hline \multicolumn{4}{|l|}{ Geopolitical zone } \\
\hline North central & 4614 & $156(13.12)$ & $33.8(28.7-39.4)$ \\
\hline North east & 6517 & $253(21.28)$ & $38.8(34.2-43.7)$ \\
\hline North west & 9096 & $414(34.82)$ & 45.5(41.3.50.0) \\
\hline South east & 2816 & $112(9.42)$ & $39.8(32.9-47.6)$ \\
\hline South south & 3747 & 114(9.59) & $30.42(25.1-36.4)$ \\
\hline $\begin{array}{l}\text { South west } \\
\text { Wealth index }\end{array}$ & 3882 & $140(11.77$ & $36.1(30.4-42.4)$ \\
\hline \multicolumn{4}{|l|}{ Wealth index } \\
\hline Poor & 14462 & $621(52.23)$ & $42.9(39.6-42.6)$ \\
\hline Average & 6272 & $227(19.09)$ & $36.2(31.7-38.7)$ \\
\hline Rich & 10748 & $341(28.68$ & $31.7(28.5-40.1)$ \\
\hline \multicolumn{4}{|c|}{ NEONATAL FACTORS } \\
\hline Male & 15965 & $683(57.44)$ & $42.7(39.7-46.0)$ \\
\hline Female & 15517 & $506(42.56)$ & $32.6(29.9-35.5)$ \\
\hline \multicolumn{4}{|l|}{ Size of the child } \\
\hline Large & 13589 & $378(31.79)$ & $27.8(1.4-30.7)$ \\
\hline Average & 15976 & $621(52.23)$ & $38.9(1.5-41.9)$ \\
\hline Small & 1917 & $190(15.98)$ & $99.1(6.8-113)$ \\
\hline $\begin{array}{l}\text { Birth Order } \\
1\end{array}$ & 6109 & $286(24.05)$ & $46.8(41.7-52.4)$ \\
\hline $2-3$ & 10074 & $322(27.08)$ & $42.0(28.6-35.6)$ \\
\hline $4-6$ & 9971 & $327(27.50)$ & $32.8(29.4-36.5)$ \\
\hline$\geq 7$ & 5328 & $254(21.36)$ & $47.7(42.1-53.7)$ \\
\hline
\end{tabular}


Table 4: Association between the Utilization of Maternal Health Services and Neonatal Mortality

\begin{tabular}{|c|c|c|c|c|}
\hline \multirow[t]{2}{*}{ Continuum of Maternal Care } & \multicolumn{2}{|c|}{ Neonatal Mortality } & \multirow[b]{2}{*}{$\mathrm{Chi}^{2}$} & \multirow[b]{2}{*}{$P$ value } \\
\hline & Present (\%) & Absent (\%) & & \\
\hline \multicolumn{5}{|l|}{ Antenatal Care } \\
\hline ANC by skilled provider & 51.4 & 41.2 & 30.2 & $<0.001$ \\
\hline At Least (4) Four ANC Visit & 50.4 & 49.6 & 2.9 & 0.084 \\
\hline Told Complications & 53.9 & 46.1 & 34.3 & $<0.001$ \\
\hline \multicolumn{5}{|l|}{ Delivery Care } \\
\hline Skilled Attendance at Birth & 53.0 & 47.0 & 55.6 & $<0.001$ \\
\hline Caesarean Section & 78.7 & 21.3 & 36.1 & $<0.001$ \\
\hline \multicolumn{5}{|l|}{ Postnatal Care } \\
\hline PNC by skilled provider & 56.3 & 43.7 & 30.7 & $<0.001$ \\
\hline $\begin{array}{l}\text { Initiate } \quad \text { Breastfeeding } \\
\text { immediately }\end{array}$ & 34.2 & 65.8 & 6.3 & 0.012 \\
\hline
\end{tabular}

Table 5: Hazards ratios (HR) and 95\% confidence interval (Cl) from multilevel Cox proportional hazard regression analysis showing the effects of ANC and selected variables on neonatal mortality in Nigeria

\begin{tabular}{|c|c|c|c|c|}
\hline Characteristics & Model 0 & Model 1 & Model 2 & Model 3 \\
\hline Fixed Effects & Empty Model & $\mathrm{HR}(\mathrm{Cl})$ & $\mathrm{HR}(\mathrm{Cl})$ & $\mathrm{HR}(\mathrm{Cl})$ \\
\hline \multicolumn{5}{|l|}{ Antenatal care } \\
\hline Less than 4 visits (RC) & & 1 & 1 & 1 \\
\hline $\begin{array}{l}4 \text { or more visits } \\
\text { Age Group }\end{array}$ & & $0.76(0.61-0.95)^{*}$ & $0.80(0.66-0.97)^{*}$ & $0.78(0.61-0.98)^{*}$ \\
\hline $15-24(\mathrm{RC})$ & & 1 & & 1 \\
\hline $25-34$ & & $1.78(1.29-2.47)^{\star}$ & & $1.81(1.31-2.51)^{*}$ \\
\hline $35+$ & & $3.08(1.99-4.76)^{*}$ & & $3.15(2.04-4.88)^{\star}$ \\
\hline \multicolumn{5}{|l|}{ Age at birth } \\
\hline $15-24(\mathrm{RC})$ & & 1 & & 1 \\
\hline $25-34$ & & $1.00(0.43-2.35)$ & & $0.99(0.42-2.33)$ \\
\hline \multicolumn{4}{|l|}{ Religion } & $1.55(0.62-3.88)$ \\
\hline Christianity (RC) & & 1 & & 1 \\
\hline Islam & & 0.87 (0.69-1.09) & & $0.81(0.58-1.13)$ \\
\hline Others & & $1.26(0.58-2.74)$ & & $1.29(0.59-2.80)$ \\
\hline \multicolumn{5}{|l|}{ Birth Interval } \\
\hline <24 months (RC) & & 1 & & 1 \\
\hline 24-35 months & & $0.73(0.57-0.94)^{*}$ & & $0.75(0.58-0.96)^{*}$ \\
\hline $36+$ & & $0.61(0.47-0.79)^{*}$ & & $0.63(0.49-0.81)^{*}$ \\
\hline \multicolumn{5}{|l|}{ Parity } \\
\hline$<3(\mathrm{RC})$ & & 1 & & 1 \\
\hline $3-4$ & & $0.37(0.29-0.48)^{*}$ & & $0.37(0.29-0.47)^{*}$ \\
\hline $\begin{array}{l}5+ \\
\text { Wealth Index }\end{array}$ & & $0.25(0.19-0.33)^{\star}$ & & $0.24(0.180 .32)^{*}$ \\
\hline \multicolumn{5}{|l|}{ Wealth Index } \\
\hline Poor (RC) & & 1 & 1 & 1 \\
\hline Middle & & $0.92(0.70-1.21)$ & $1.16(0.92-1.46)$ & $0.94(0.69-1.27)$ \\
\hline Rich & & $0.76(0.58-1.00)^{*}$ & $1.33(1.04-1.69)^{*}$ & $0.90(0.64-1.26)$ \\
\hline \multicolumn{5}{|l|}{ Marital Status } \\
\hline Never married (RC) & & 1 & & 1 \\
\hline Currently married & & $1.00(0.32-3.16)$ & & 1.04 (0.33-3.29) \\
\hline Previously married & & 0.85 (0.22-3.33) & & $0.87(0.22-3.42)$ \\
\hline \multicolumn{5}{|l|}{ Region } \\
\hline South-west (RC) & & & 1 & 1 \\
\hline North Central & & & $0.82(0.57-1.16)$ & 0.89 (0.58-1.35) \\
\hline North East & & & $1.08(0.77-1.50)$ & $1.19(0.77-1.83)$ \\
\hline North West & & & $0.93(0.66-1.30)$ & $1.12(0.72-1.74)$ \\
\hline South East & & & $1.47(1.03-2.10)^{*}$ & 1.35 (0.87-2.09) \\
\hline South South & & & $1.04(0.73-1.48)$ & 1.06 (0.70-1.62) \\
\hline \multicolumn{5}{|l|}{ Place of Residence } \\
\hline Urban (RC) & & & 1 & 1 \\
\hline Rural & & & $1.34(1.08-1.66)^{*}$ & $1.44(1.09-1.90)^{*}$ \\
\hline \multicolumn{5}{|l|}{ Community Poverty } \\
\hline Rich (RC) & & & & 1 \\
\hline Middle & & & & 1.09 (0.83-1.42) \\
\hline Poor & & & & 1.32 (0.99-1.78) \\
\hline
\end{tabular}

http://aps.journals.ac.za 


\begin{tabular}{|c|c|c|c|c|}
\hline \multicolumn{5}{|c|}{ Community level of Education } \\
\hline Low $(\mathrm{RC})$ & & & 1 & 1 \\
\hline Medium & & & $1.17(0.91-1.50)$ & $1.11(0.81-1.51)$ \\
\hline High & & & $0.97(0.73-1.29)$ & $0.90(0.63-1.30)$ \\
\hline Random Effects & Null Model & Model 1 (ANC) & Model 2 & Model 3 \\
\hline \multicolumn{5}{|l|}{ Community-Level } \\
\hline $\begin{array}{l}\text { Variance (SE) } \\
\text { Model fit statistics }\end{array}$ & $0.73(0.12)^{*}$ & $0(0.01)$ & $0(0.01)$ & $0(0.01)$ \\
\hline AIC & 10084.7 & 3763.8 & 5166.7 & 3768.8 \\
\hline $\mathrm{BIC}$ & 10109.8 & 3902.2 & 5277.5 & 3984.1 \\
\hline
\end{tabular}

${ }^{*} \mathrm{p}<0.05, \mathrm{SE}=$ Standard Error, $\mathrm{AIC}=$ Akaike information criterion, $\mathrm{BIC}=$ Bayesian information criterion

Table 6: Hazard ratio (HR) and 95\% confidence interval (Cl) from multilevel Cox proportional hazard regression analysis showing the effects of PNC and selected variables on neonatal mortality in Nigeria

\begin{tabular}{|c|c|c|c|c|}
\hline Characteristics & Model 0 & Model 1 & Model 2 & Model 3 \\
\hline Fixed Effects & Empty Model & $\mathrm{HR}(\mathrm{Cl})$ & $\mathrm{HR}(\mathrm{Cl})$ & $\mathrm{HR}(\mathrm{Cl})$ \\
\hline \multicolumn{5}{|l|}{ Postnatal care } \\
\hline \multicolumn{2}{|l|}{ Had no PNC (RC) } & 1 & 1 & 1 \\
\hline \multirow{2}{*}{\multicolumn{2}{|c|}{ provider }} & $0.40(0.29-0.54)^{*}$ & $0.35(0.27-0.47)^{*}$ & $0.41(0.30-0.56)^{*}$ \\
\hline \multicolumn{4}{|l|}{ Age Group } & \\
\hline \multicolumn{2}{|l|}{$15-24(\mathrm{RC})$} & 1 & & 1 \\
\hline \multicolumn{2}{|l|}{$25-34$} & $1.95(1.39-2.73)^{*}$ & & $1.98(1.41-2.78)^{*}$ \\
\hline \multirow{2}{*}{\multicolumn{2}{|c|}{$\begin{array}{l}35+ \\
\text { Age at birth }\end{array}$}} & $3.34(2.15-5.33)^{*}$ & & $3.46(2.20-5.44)^{*}$ \\
\hline & & & & \\
\hline \multicolumn{2}{|l|}{$15-24(R C)$} & 1 & & 1 \\
\hline \multicolumn{2}{|l|}{$25-34$} & $1.01(0.40-2.56)$ & & $1.01(0.40-2.55)$ \\
\hline \multirow{2}{*}{\multicolumn{5}{|c|}{$1.51(0.56-4.08)$}} \\
\hline & & & & \\
\hline \multicolumn{2}{|l|}{ Christianity (RC) } & 1 & & 1 \\
\hline \multicolumn{2}{|l|}{ Islam } & $0.88(0.70-1.11)$ & & $0.80(0.56-1.13)$ \\
\hline \multirow{2}{*}{\multicolumn{5}{|c|}{$\begin{array}{l}\text { Others } \\
\text { Birth Interval }\end{array}$}} \\
\hline & & & & \\
\hline \multicolumn{2}{|l|}{$<24$ months $(\mathrm{RC})$} & 1 & & 1 \\
\hline \multicolumn{2}{|l|}{$24-35$ months } & $0.81(0.62-1.05)$ & & $0.82(0.63-1.06)$ \\
\hline \multicolumn{2}{|l|}{$36+$} & $0.65(0.50-0.84)^{*}$ & & $0.66(0.50-0.86)^{*}$ \\
\hline \multicolumn{5}{|l|}{ Parity } \\
\hline \multicolumn{2}{|l|}{$<3(\mathrm{RC})$} & 1 & & 1 \\
\hline \multicolumn{2}{|l|}{$3-4$} & $0.38(0.29-0.49)^{*}$ & & $0.38(0.29-0.48)^{*}$ \\
\hline \multirow{2}{*}{\multicolumn{2}{|c|}{$\begin{array}{l}5+ \\
\text { Wealth Index }\end{array}$}} & $0.24(0.18-0.33)^{*}$ & & $0.24(0.18-0.32)^{*}$ \\
\hline & & & & \\
\hline \multicolumn{2}{|l|}{ Poor (RC) } & 1 & & 1 \\
\hline Middle & & $0.86(0.65-1.15)$ & & $0.89(0.65-1.22)$ \\
\hline Rich & & $0.86(0.66-1.12)$ & & $1.00(0.71-1.41)$ \\
\hline Marital Status & & & & \\
\hline Never married (RC) & & 1 & & 1 \\
\hline Currently married & & $0.85(0.27-2.70)$ & & $0.85(0.27-2.71)$ \\
\hline Previously married & & $0.69(0.17-2.80)$ & & $0.69(0.17-2.78)$ \\
\hline Region & & & & \\
\hline South-west (RC) & & & 1 & 1 \\
\hline North Central & & & $0.72(0.50-1.04)$ & $0.81(0.53-1.25)$ \\
\hline North East & & & 0.89 (0.63-1.26) & $1.08(0.70-1.69)$ \\
\hline North West & & & $0.76(0.53-1.07)$ & $1.00(0.63-1.57)$ \\
\hline South East & & & $1.08(0.74-1.59)$ & $1.03(0.65-1.64)$ \\
\hline South South & & & $0.83(0.57-1.20)$ & $0.88(0.57-1.37)$ \\
\hline Place of Residence & & & & \\
\hline Urban (RC) & & & 1 & 1 \\
\hline Rural & & & $1.28(1.02-1.61)^{*}$ & $1.43(1.07-1.90)^{*}$ \\
\hline Community Poverty & & & & \\
\hline Rich (RC) & & & 1 & 1 \\
\hline Middle & & & $1.16(0.91-1.48)$ & $1.11(0.84-1.46)$ \\
\hline Poor & & & $1.30(1.00-1.68)^{*}$ & $1.30(0.96-1.76)$ \\
\hline Community level of Educa & ion & & & \\
\hline Low $(\mathrm{RC})$ & & & 1 & 1 \\
\hline Medium & & & $1.17(0.91-1.51)$ & $1.10(0.80-1.51)$ \\
\hline High & & & $1.06(0.79-1.41)$ & $0.89(0.62-1.30)$ \\
\hline
\end{tabular}




\begin{tabular}{lllll}
$\begin{array}{l}\text { Random Effects } \\
\text { Community-Level }\end{array}$ & Empty & Model 1 (PNC) & Model 2 & Model 3 \\
\hline $\begin{array}{llll}\text { Variance (SE) } \\
\text { Model fit statistics }\end{array}$ & $0.73(0.12)^{*}$ & $0(0.01)$ & $0(0.01)$ & $0(0.01)$ \\
AlC & 10084.7 & 3548.5 & 4849.1 & 3556.8 \\
BIC & 10109.8 & 3686.2 & 4959.4 & 3771.2 \\
\hline${ }^{*}$ p $<0.05$, SEEStandard &
\end{tabular}

${ }^{*} \mathrm{p}<0.05, \mathrm{SE}=\mathrm{Standard}$ Error, $\mathrm{AIC}=$ Akaike information criterion, $\mathrm{BIC}=$ Bayesian information criterion

\section{Discussion and Conclusion}

In this study we examined the influence of maternal health care utilization on neonatal survival in Nigeria. The study explored the pathway of influence through which maternal health care affects neonatal survival, beyond the factors that exist at the individual level. Although, neonatal mortality is a neglected public health challenge in Nigeria, it accounts for the largest number of infant deaths in the country, with 37 neonatal deaths per 1000 live births out of a total of 69 infant deaths per 1000 live births (Adedini et al, 20I5b; NPC \& ICF International, 20I4). Persistence of high neonatal mortality in Nigeria contributed to the country's failure to achieve the MDG4, which sought to reduce under 5 mortality to two-thirds between 1990 and 2015 (UNDP, 2015). Meanwhile, majority of the causes of neonatal death are preventable through simple and cost-effective interventions which can be delivered through maternal health services in continuum of care from pregnancy to the immediate post-delivery period, including services such as antenatal care, delivery care and postnatal care (Darmadt, 2005). Hence, this study examined the relationship between the utilization of maternal health services and neonatal mortality in Nigeria by carrying out a multilevel analysis of the most recent Nigeria Demographic and Health Survey (NDHS 20I3) data of women.

Importantly, we found that women who had 4 or more antenatal visits by skilled providers had significantly lower risk of neonatal death $(H R=0.78)$ compared with those who had less number of visits. This finding is supported by studies from Indonesia (Titaley et al, 2008), Pakistan (Nisar et al, 20l4) and Ghana (Kayode $\mathrm{G}$ et al, 20/4). Mizumuto and colleagues (2015) in Brazil found that neonates born to mothers with inadequate number of antenatal visits were at a higher risk for early neonatal infections compared with controls. However, this is against the backdrop that high ANC coverage alone (measured as having had at least one ANC visit) had not resulted into improved maternal and birth outcomes (Boller, 2003; Babalola S \& Fatusi, 2009). Therefore, there has been a shift in emphasis from the sheer number of ANC visits to the quality of antenatal care particularly the type of interventions obtained during such visits (Sarker, 2010; Tetui et al, 20I3). Hence, the WHO (20I3) now recommends four antenatal as the optimum number of visits required to obtain the needed interventions that can tackle the risk factors of neonatal death in the mother. These include Intermittent Preventive Treatment for malaria (IPT), tetanus toxoid immunization, prevention of mother to child transmission, identification and management of preeclampsia and so on. Furthermore, it is necessary to tailor the range of services provided to the assessed need of the particular population. For example, Singh et al (2013) documented that obtaining TT toxoid by mothers during their ANC visit was the most protective antenatal care interventions in improving neonatal survival in India. This finding suggest that the adaption policies and framework targeted at neonatal mortality reduction as the WHO Maternal, Newborn and Child policy (PMNCH 20I5) ought to be based on the epidemiologic profile and the perceived effectiveness of the diverse interventions in the various different populations.

Our study also showed the protective effect of postnatal care on neonatal survival, even after adjusting for the effects of selected individual- and community-level variables. Although, postnatal had been the most neglected in the range of maternal health care services, it has recently begun to receive attention as a crucial strategy in reducing neonatal deaths. This is because majority of these deaths occur in the first day and first week of life. This underscores the need to provide postnatal care to women and their newborns in the critical period when the risk of death is highest. Singh et al (20/2) in a nationally representative data in India showed that the place of postnatal check was important to neonatal survival as mothers that obtained PNC from government facilities were better protected that those had no PNC at all. The WHO recommends that postnatal checks should be provided within the first $24-48$ hours in order to detect danger signs in the newborn, to initiate breastfeeding early, provide clean cord care and maintain temperature by the management of hypothermia and Kangaroo care (WHO, 20I3). Although PNC from skilled worker is preferred, obtaining care from an unskilled worker was better than no care at all. Singh and co-workers (2014) using DHS data from 10 African countries investigated the associations between the PNC and NNM after controlling for relevant variables using multilevel analysis. They found that PNC whether provided by skilled and unskilled provider was better than no 
PNC at all. They argued that while PNC by skilled provider is the standard, PNC by unskilled provider might be a short-term approach because they could be linked to the skilled PNC by a strong referral system (Singh et al, 20I2).

In this study, we examined the association between skilled attendance and NNM using bivariate analysis and not multivariate analysis. We found that skilled attendance was significantly associated in NNM. Generally skilled attendance at birth is expected to result in improved neonatal outcomes. Although few studies have examined the association skilled birth attendance at birth with NNM using nationally representative data. Singh and co-workers (2014) investigated the consistency of skilled attendance at birth in decreasing neonatal mortality across 3 continents of Africa, Asia and Latin American and the Caribbean using the most recent Demographic Health Survey data from 9 countries. Unexpectedly, SA was not associated with decreased in neonatal mortality overall. However when disaggregated by continent, SA was associated with a decline in neonatal mortality in Latin America while it was associated with a rise in Africa. They argued that the reason for adverse neonatal outcome in Africa is because SA have varying levels of skills and competence and are challenged by poor health infrastructure at the same time they supervise more complicated deliveries which had been mismanaged by unskilled health workers.

Even though, our study showed the importance of antenatal care and postnatal care services for the survival of newborns, our findings further suggested the need to look beyond the micro-level pathway of influence, including the social determinants of health in the efforts to increase the utilization of these important public health interventions in Nigeria. Social determinants of health, defined as 'the conditions in which people are born, grow, live, work and age, and the systems put in place to prevent disease and treat illness' (CSDH 2008 pp 42.), predicts the highest level variations in health outcomes. Generally, we also found that neonatal mortality rates were higher among older women, those with lower level of education, higher parity, short birth intervals, those living in poverty, the unmarried, rural residence and those in the northern Nigeria. Therefore, these factors need to be addressed in the implementation of programmes that target neonatal survival at the population level. Moreover, we further explored the contextual issues influencing neonatal mortality which have not been sufficiently investigated in Nigeria. Notably, community-level factors have been shown to be associated with neonatal mortality and morbidity (Chowdhury QH et al 2010; Mekonnen et al 2015.)
Van de poel et al (2009) in their study of the household and community characteristic of infant mortality in francophone-Africa emphasized the importance of decomposing the determinants of infant mortality at different levels in order to identify the critical point for public health policy and intervention.

The place of residence was the most significant factor associated with neonatal mortality of the community level factors examined. Women living in rural communities had about $30-40 \%$ risk of experiencing neonatal death compared to urban dwelling women. The rural- urban differences in neonatal mortality have reported in some countries including Nigeria. Adewuyi and Zhao (2017) in a recent study in which the determinants of neonatal mortality was disaggregated by rural and urban residence, found that low birth weight and short birth interval key determinants of NND in rural Nigeria. In addition, the NDHS 2013 revealed that rural dwellers were more likely to deliver at home, less likely to obtain the complete doses of tetanus toxoid immunization, to have skilled attendance at birth and receive postnatal care. This explains for instance why neonatal tetanus (NNT) is the leading cause of NNM in communities where delivery takes place predominantly at home (Singh 2013). Yi and coworkers (20I I) reported that the drivers of adverse neonatal outcomes in rural areas of China were poverty, illiteracy, and unemployment. And resulted in higher NNM rates through the lack of mother education, lack of access to health care services, high and unaffordable health care costs and lack of quality neonatal care. The programmatic implications of some of the challenges in the rural areas are documented in Africa's policy framework for promoting neonatal survival which postulates that the implementation essential interventions can lead to $90 \%$ reduction in the burden of neonatal mortality within the continent. These programmes include strengthening national policies and developing locally adapted guidelines, strengthening the quality of maternal health services by training of staff in newborn care and resuscitation, providing equipment, essential drugs logistics and supplies. More importantly is the need to remove the barriers against the utilization of maternal health services particularly in the rural areas which include user fees, long travel distance and addressing poor staff attitude by having more government investment into neonatal health (Disu, 20I0).

The study concludes that the pathway of influence through which maternal health care influences neonatal survival goes beyond the factors that exist at the individual level. Community contextual factors also play significant roles in influencing maternal 
health care utilization. Hence, this study suggests the need to address contextual barriers to maternal health care utilization in the programmatic efforts towards achieving neonatal mortality reduction in Nigeria.

\section{Limitations of study}

One limitation of the study is the adoption of primary sampling units (PSUs) as a proxy for community. This may lead to some bias as a result of misclassification of respondents into wrong administrative boundaries. Another limitation is the inability to explore contextual factors that address community values and norms related to child care and health care uptake in different communities. The temporal sequence of events for some characteristics may also pose some limitation. Notwithstanding these limitations, the study provides useful insights about the pathway of influence regarding the relationship between maternal health care utilization and neonatal mortality in Nigeria.

\section{REFERENCES}

Acuin, C.S, Khor G.L., Liabsuetrakul.T., Achadi, E.L., Htay, T.T., Firestone R., Bhutta, Z.A. 20II Maternal, neonatal, and child health in Southeast Asia; towards greater regional collaboration; Lancet: 377; 516-25.

Adedini S.A, Odimegwu C., Imasiku, E.N.S Ononokpono,D. 2015. Ethnic differentials in under-five mortality in Nigeria Ethnicity \& Health, Vol. 20, No. 2, 145-162, http://dx.doi.org// 0.1080// 3557858.20|4.890599

Adewuyi, E.A., and Yun, Zhao. 2017. Determinants of neonatal mortality in rural and urban Nigeria: Evidence from a population-based national survey. Pediatrics International, Volume 59, Issue 2 February 2017 Pages 190-200

Akinyemi, J.O., Elijah Afolabi Bamgboye, E.A., and Ayeni O. 2015 Trends in neonatal mortality in Nigeria and effects of bio-demographic and maternal characteristics. BMC Pediatrics (I5:36 DOI I0.1I86/s I2887-0I5-0349-0. Assessed 13th February 2016

Antai, D. 20II. "Inequalities in Under-5 Mortality in Nigeria: Do Ethnicity and Socioeconomic Position Matter?" Journal of Epidemiology 2I (I): I3-20. doi:I0.2I88/jea.JE20100049.

Babalola, S., and Fatusi. 2009 A Determinants of use of maternal health services in Nigeria - looking beyond individual and household factors BMC Pregnancy Childbirth. Sep 15; 9:43. doi: I0. I 186/ |47|-2393-9-43

Boller, C., Wyss, K., Mtasiwa, D., Tanner, M. 2003. Quality and comparison of antenatal care in public and private providers in the United Republic of
Tanzania Bull World Health Organ. vol.8I n.2 Geneva. $\quad$ http://dx.doi.org//0.1590/S004296862003000200008

Chowdhury, M.E., Akhter, H.H., Virasakdi, C., Alan, F., Geater, A.F. 2005. Neonatal Mortality in Rural Bangladesh: An Exploratory Study J Health Popul Nutr. Mar; 23(I): 16-24

Chowdhury, Q.H., Islam, R., and Hossain, K. 2010. Socio-economic determinants of neonatal, post neonatal, infant and child mortality. International Journal of Sociology and Anthropology Vol. 2(6), pp. II 8-I.

$\mathrm{CSDH}, 2008$. Closing the gap in a generation: health equity through action on the social determinants of health. Final Report of the Commission on Social Determinants of Health. Geneva, World Health Organization.

Darmstadt, G.L., Bhutta, Z.A., Cousens, S., Adam, T., Walker, N., De, Bernis, L. 2005. Evidence-based, cost-effective interventions: how many newborn babies can we save? Lancet. 2005 Mar 12-18; 365(9463):977-88

Ezeh, O.K., Agho, K.E., Dibley, M.J., Hall, J., and Page, A.N. 20l4. Determinants of neonatal mortality in Nigeria: evidence from the 2008 demographic and health survey BMC Public Health 2014, 14:521

Disu E Challenges of Neonatal Care in Nigeria: What Solutions for Child Survival

Kolola, T., Ekubay, M., Tesfa, E., Morka, W. (20I6). Determinants of Neonatal Mortality in North Shoa Zone, AmRegional State, Ethiopia. PLoS ONE II(I0): e0I64472. doi:I0.137I/pone.0164472

Kayode, G.A., Ansah, E., Akua, I., Agye, pong, Mary, Amoakoh-Coleman, Diede rick, E., Grobbee, and Kerstin, K., lipstein- Grobusch. 2014.: Individual and community determinants of neonatal mortality in Ghana: a multi-level analysis BMC Pregnancy and Childbirth, 14:165.

Lawn, J., Kerber, K., Enweronu-Laryea, C., Massee, Bateman, O. 2009. Newborn survival in low resource settings-are we delivering? 'BJOG 16 (Suppl. I):49-59.

Lawn, J.E., Cousens, S., Zupan, J. 2005: Four million neonatal deaths: when? Where? Why? Lancet 365:89I-900, 2005

Liu L., Johnson H.L., Cousens S., Perin J.,

Scott S., Lawn J.E., et al 2012; Global, regional, and national causes of child mortality: an updated systematic analysis for 2010 with time trends since $2000 \quad$ Lancet 379: 2I5I-6I http://dx.doi.org/I0.10I6/S0 I40-6736(I2)60560-I Marsh, D.R., Darmstadt, G.L., Moore, J., Daly, P., Oot, D., Tinker, A. 2002. Advancing Newborn Health and Survival in Developing Countries: A 
Conceptual Framework: Journal of Perinatology 22, 572-576 doi: I0.1038/sj.jp.721 0793

Martines, J., Paul, V.K., Bhutta, Z.A., Koblinsky, M., Soucat, A., Walker, $N$ et al. 2005. Neonatal survival: a call for action. Lancet; 365: I I 89-97

Mwansa-Kambafwile, J., Cousens, S., Hansen, T., Lawn, J.E. 2010. Antenatal steroids in preterm labour for the prevention of neonatal deaths due to complications of preterm birth. Int J Epidemiol. 2010 Apr; 39 Suppl 1:il22-33. doi: 10.1093/ije/dyq029.

Mekonnen, Tensou, B., Telake, D., Degefie, T., and Bekele, A. 2013. Neonatal mortality in Ethiopia: trends and determinants BMC Public Health, 13:483 http://www.biomedcentral.com/I47| 2458/I 3/483

Mizumotoa, B.R., Moreiraa, B.M., Santoro-Lopesa, G., Cunhae, A.J., dos Santosf RMR, Pessoa-Silvag, C.L. 2015. Quality of antenatal care as a risk factor for early onset neonatal infections in Rio de Janeiro, Brazil b r a z j i n f e c t d i s.; 9 9(3):272277

Mosley, W., and Chen, L. C. 1984. An analytical framework for the study of child survival in developing countries. Population and Development Review 10.suppl:25-45

Nigeria Demographic and Health Survey 2013. National Population Commission and ORC Macro International, Calverton MD.

Nisar, Y.B., and Dibley, M.J. 2014Determinants of neonatal mortality in Pakistan: secondary analysis of Pakistan Demographic and Health Survey 2006-07 BMC Public Health, 14:663 http://www.biomedcentral.com/ I47I2458/I4/663

Omariba, W.R., Beaujot,R. Rajulton F. 2007. Determinants of infant and child mortality in Kenya: an analysis controlling for frailty effects Popul Res Policy Rev 26:299-32I DOI |0.|007/s | | | |3-007-903 |-z

Oesterggaard, M.Z., Inoue, M., Yoshida, S., Mahanani, W.R., Gore, F.M, Cousens, S., et al. 201I. Neonatal mortality levels for 193 countries in 2009 with trends since 1990: A systematic analysis of progress, projections, and priorities. PLoS Med. 201I; doi:I0.137|/journal.pmed. I00I080.

Owa J.A. \& Osinaike A.I. 1998. Neonatal morbidity and mortality in Nigeria. The Indian Journal of Pediatrics May 1998, Volume 65, Issue 3, pp 44I449

PMNCH Progress Report 2015 Achieving the world we want for women children and adolescents "Developing strategies to deliver the 2030 agenda" The Partnership for Maternal, Newborn and Child Health
Sarker, M., Schmid, G., Larsson, E., Kirenga, S., De, Allegri, M., Neuhann, F., Mbunda, T., Isaack, Lekule, I., and Müller, O. 20I0. Quality of antenatal care in rural southern Tanzania: a reality check. BMC Research Notes 3:209 doi: 10.1 I86/1756-0500-3-209

Singh,K. Brodish. P. Haney E. 20I4: Postnatal care by provider type and neonatal death in sub-Saharan Africa: a multilevel analysis. BMC Public Health |4:94 I. doi:|0. I |86/|47|-2458-|4-94 |

Singh, A., Yadav, A., Ashish Singh, A. 2012 Utilization of postnatal care for newborns and its association with neonatal mortality in India: An analytical appraisal. BMC Pregnancy and Childbirth, 12:33. doi: I0.1 I86/147|-2393-12-33

Singh, A., Kumar, A., Kumar, A. (20/3) Determinants of neonatal mortality in rural India, 2007-2008. Peer J I:e75 https://doi.org//0.77/ 7/peerj.75

Singh, K., Brodish, P., Suchindran, C. A. 2014. Regional Multilevel Analysis: Can Skilled Birth Attendants Uniformly Decrease Neonatal Mortality? Matern Child Health J. Jan; 18(I):2429. doi: I0. I007/s 10995-0I3-I260-7

Singh, A., Pallikadavath, S., Ram, F., Alagarajan, M. 2014. Do antenatal care interventions improve neonatal survival in India? Health Policy Plan 29 (7): 842-848. DOI: https://doi.org/10.1093/heapol/czt066

Shiffman, J. 2010. Issue attention in global health: the case of newborn survival. Lancet 375: 2045-2049.

Suleiman, M.B., Mokuolu, O.A. 2014. Perinatal mortality in a northwestern Nigerian city: a wakeup call. Frontiers in Pediatrics Neonatology, Volume2; 105

Titaley, C.R., Dibley, M.J., AghoK, Roberts, C. L., Hall, J. 2008: Determinants of neonatal mortality in Indonesia. BMC Public Health, 8:232.

UNDP. Nigeria MDGs Performance Survey Report. 2015.

www.ng.undp.org/content/nigeria/en/.../mdg/Nige riaMDGsSurveyReport2015.html

Van, De, Poel, E., O'donnell, O., and Van, Doorslaer, A.E. 2009. What Explains the Rural-Urban Gap in Infant Mortality: Household or Community Characteristics? Demography Volume 46-Number 4, 827-850

Winch, P. J., Alan, M. A., Akther, A., Afroz, D., Ali, N. A., Ellis, A. A. et al. 2005. Local understanding of vulnerability and protection during the neonatal period in Sylhet District, Bangladesh: a qualitative study. Lancet; 366: 478-85.

World Health Organization (WHO) 1996. Perinatal Mortality three-quarter. A Listing of Available Information. Maternal Health and Safe 
Motherhood Programme Geneva: World Health Organization (WHO), (WHO/FRM/MSM/96.7)

WHO. World Health Report 2005. Make every mother and child count. 2005. Geneva Switzerland, WHO.

World Health Organization. 2013. WHO recommendations on postnatal care of the mother and newborn www.who.int/maternal_child_adolescent/docume nts/postnatal-care.../en/ Accessed 20 May 2016. p. 9-12.

United Nations Children Fund (UNICEF) 20II. Levels and Trends in Child Mortality Report 201 I generated by UN Inter-agency group on Childhood mortality estimation.http://www.childinfo.org/files/Child_M ortality_Report_20II.pdf. Accessed 20 May 2016. p. 9-I2.

Yi, B., Wu, L., Liu, H., Fang, W., Hu, Y., and Youjie, W. 20II. Rural-urban differences of neonatal mortality in a poorly developed province of China. BMC Public Health 20 I I:477

Integrated maternal newborn and child health (imnch) strategy - Unicef

https://www.unicef.org/nigeria/ng_publications_IMN CHbrochure.pdf. Accessed $21^{\mathrm{st}}$ March 2017. 